A. Brömme, C. Busch, A. Dantcheva, C. Rathgeb and A. Uhl (Eds.): BIOSIG 2018, Lecture Notes in Informatics (LNI), Gesellschaft für Informatik, Bonn $2018 \quad 1$

\title{
Technology Gap Navigator: Emerging Design of Biometric-Enabled Risk Assessment Machines
}

\author{
Shawn Eastwood ${ }^{1}$ Ken Lai $^{2}$ Svetlana Yanushkevich ${ }^{3}$ Richard Guest ${ }^{4}$ Vlad Shmerko ${ }^{5}$
}

\begin{abstract}
This paper reports the Technology Gap (TG) navigator, a novel tool for individual risk assessment in the layered security infrastructure. It is motivated by the practical need of the biometricenabled security systems design. The tool helps specify the conditions for bridging the identified TGs. The input data for the TG navigator includes 1) a causal description of the TG, 2) statistics regarding the available resources and performances, and 3) the required performance. The output includes generated probabilistic conditions, and the corresponding technology requirements for bridging the targeted TG.
\end{abstract}

Keywords: technology gap, causal model, biometrics, risks

\section{Introduction and motivation}

Technology Gap (TG) navigation is a mechanism for 1) the analysis of the difference between required and available technologies, and 2) generating certain conditions for bridging this TG, given a design scenario or a task. For example, a resent study [GNQ17] addresses the state-of-the-art in the identification of non-cooperative individuals, such as surveillance in mass-transit systems. Another example of a TG [Nu14] involves the operational performance of biometric-based recognition in border control systems, which is significantly lower than a "theoretical" performance. This scenario can be represented by a model at a reasonable level of abstraction and conditions for filling or bridging this gap can be found. This is the core idea of TG navigation, and emerging design approaches.

In this paper, we provide an example of TG navigation for designing a future generation of biometric-enabled risk assessment machines. A particular case of such machines is known as Automated Border Control machines [Do16]. The TG related problems include 1) TG identification, and 2) TG bridging. In particular, approaches to the TG analysis at a high level of abstraction include the following:

- Smart Border doctrine [EU14]. The TG is identified by a set of indicators from the field of biometrics, decision-making, intelligent computing, risk assessment, and privacy protection. In the TG analysis of the checkpoint of the future [In14], the basic TG drivers are defined in terms of traveler risk assessment, identity management, behavior analysis, and alternative measures for unpredictability.

\footnotetext{
${ }^{1}$ Biometric Technologies Laboratory, ECE Department, University of Calgary, Canada, sceastwo@ucalgary.ca

${ }^{2}$ Biometric Technologies Laboratory, ECE Department, University of Calgary, Canada, kelai@ucalgary.ca

${ }^{3}$ Biometric Technologies Laboratory, ECE Department, University of Calgary, Canada, syanshk@ucalgary.ca

${ }^{4}$ School of Engineering and Digital Arts, University of Kent, UK, r.m.guest@kent.ac.uk

${ }^{5}$ Biometric Technologies Laboratory, ECE Department, University of Calgary, Canada, vshmerko@ucalgary.ca
} 
- Public security. In a layered security concept [CHR15], the TG addresses an optimal distribution of layers over limited resources. Predictability is a cornerstone of public security [Li14]. The TG analysis aims at the reliable prediction of various actions.

Bridging the TGs is a challenging problem that has been studied in the following areas:

- $\quad$ forensics and biometrics [JR15];

- $\quad$ forensics and biometric-enabled watchlist screening [ANH18, La17];

- deception and biometrics [Ab17];

- $\quad$ balancing privacy, security, and cost [EU14].

Examples of TG bridging at a low level of abstraction include the meta-analysis of attacks [Bi17]. The TGs are identified in detecting a deception in interview supporting machines [RZ14, La17]. Self-service technologies for air-travelers such as reservation and payment for tickets online, checking-in over the Internet or mobile phones, picking up boarding passes at airport kiosks, and baggage drop-off [KS18] are used as additional sources for traveler risk assessment. Some formalized approaches are based on deriving the TG measures in terms of categories [Pa08], as well as technological drivers [Ha05] such as: (1) Opportunistic driver (whether or not a suitable signatures can be developed), (2) Mature driver (whether or not a suitable deployment scenario can be developed), and (3) Development driver (whether or not a suitable measurement method can be developed).

The TG navigator proposed in this paper explores these three drivers using the machine reasoning model known as a Bayesan Network $(\mathrm{BN})$. In our approach to biometric-enabled system design, Gap analysis involves the comparison of the actual performance with the desired one, aiming to identify steps needed to achieve the desired performance. TG anal$y$ sis addresses a difference in the performances of the available technology and the desired performance. This difference can be described in terms of indicators, or categories, as well as using probabilistic metrics (as is the case in this paper). TG template is a formalized mechanism of the TG analysis. TG navigator is a machine reasoning model that generates conditions for achieving a desired performance of a biometric-enabled system.

\section{The TG navigator as an inference engine}

A theoretical framework of the proposed TG navigator includes machine reasoning, also known as inference. We implement an inference engine as a multi-metric probabilistic causal network. For the TG analysis, we also utilize techniques adopted from sensitivity analysis [BP16], such as the partitioning of the networks and probability propagation in sub-nets [Ca17], as well as methodology of the TG analysis [Ha05]. Sensitivity analysis related to biometric system design was studied in [Le13]. It assesses a biometric model via determining the relative importance of various factors. Our approach is different from [Le13] as follows:

1. We operate at a higher level of system abstraction rather than aiming at particular "improvements".

2. We use probabilistic sensitivity analysis that assumes that information is represented in the form of probability distributions, either joint or marginal. 
3. For modeling, we use a causal network instead of a set of factors.

The essence of the proposed TG navigator is a uniform modeling platform which includes the following two components: $(a)$ a graphical representation of any given scenario in the form of a causal network, and $(b)$ a mechanism of uncertainty inference in the following metrics: probabilities, and fuzzy probabilities [Re09]. Using the probability metric and the Conditional Probability Tables (CPTs), the causal networks are referred to as Bayesian Networks.

\section{Demonstrative experiments}

Consider a scenario where a subject is to be compared against a watchlist. In Fig. 1, the TG navigator scenario is represented by a causal network where:

W: The watchlist node denotes whether or not the subject is actually on the watchlist (state $w_{1}=$ 'On List') or not (state $w_{2}=$ 'Off List').

T: The threshold node denotes a number that is assigned to a recognition system to determine whether a photo is accepted (positive) or rejected (negative). Higher thresholds yield less false acceptances but more false rejections. There are 3 thresholds in this example: $t_{1}={ }^{\prime} 0$ ', $t_{2}={ }^{\prime} 5$ ' and $t_{3}={ }^{\prime} 10$ '.

S: The decision strategy node represents the methods of combining the results of matching for multiple probe images. In the $s_{1}=\mathrm{OR}$ strategy, each probe result is returned as positive if its score is greater than the threshold, else negative. In the $s_{2}=$ 'Vote' strategy, each probe casts one positive vote if its matching score is greater than the threshold, otherwise it casts a negative vote. The $s_{3}=$ 'Average' strategy sums the scores of the entire probe set, and then divides it by the number of probes yielding an average score among the probe set. If the average score is greater than the threshold then the entire set is treated as positive.

P: The number of probes equates to the simulated number (one to five $p_{1}, p_{2}, p_{3}, p_{4}, p_{5}$ ) of snapshots of the subject.

C: The correctness of the recognition system: $c_{1}=$ 'True' defines the condition where the system correctly identifies a subject on the watchlist (true acceptance) and correctly rejects subject who are not on the watchlist (true rejection). $c_{2}=$ 'False' defines the condition where the system identifies subject who are not on the watchlist as being on it (false acceptance), and the wanted subjects as not being on the watchlist (false rejection).

M: The matching node represents authentication results. $m_{1}$ denotes the scenario where a subject is identified as being on the watchlist either correctly or incorrectly. $m_{2}$ denotes the scenario where a subject is not identified as such.

The initial data that populates the CPTs is real or near real. In particular, we used the performance statistic of automated border control reported in [Nu14], as well as approximations caused by technical solutions [EU14, Do16]. The data used for the experiment is the FRGC 2.0 database which contains 568 subjects with a total of 39,328 images. Face matching was performed using the Verilook software package from Neurotechnology. 
When probability distributions are used, the CPTs are assigned to each node as shown in Figure 1 (the CPT for node $M$ is the same as in Section 3.1, and node C CPT is not shown).

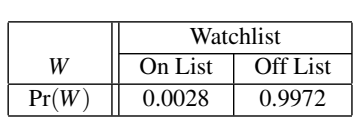

\begin{tabular}{|c||c|c|c|}
\hline \multicolumn{1}{|c||}{} & \multicolumn{3}{c|}{ Threshold } \\
\cline { 2 - 4 }$T$ & $' 0 '$ & $' 5$ & $' 10$ \\
\hline $\operatorname{Pr}(T)$ & $0 . \overline{3}$ & $0 . \overline{3}$ & $0 . \overline{3}$ \\
\hline
\end{tabular}

\begin{tabular}{|c|c|c|c|c|c|}
\hline \multirow[b]{2}{*}{$S$} & \multicolumn{4}{|c|}{ Decision Strategies } & \\
\hline & Or & Vote & \multicolumn{2}{|c|}{ Average } & \\
\hline $\operatorname{Pr}(S)$ & $0 . \overline{3}$ & $0 . \overline{3}$ & \multicolumn{2}{|c|}{$0 . \overline{3}$} & \\
\hline & \multicolumn{5}{|c|}{ Number of Probes } \\
\hline$P$ & P1 & P2 & P3 & $\mathrm{P} 4$ & P5 \\
\hline $\operatorname{Pr}(P)$ & 0.2 & 0.2 & 0.2 & 0.2 & 0.2 \\
\hline
\end{tabular}

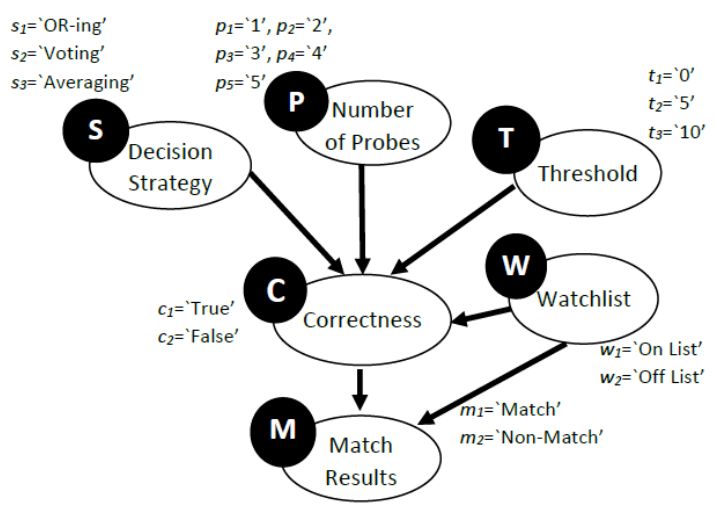

Fig. 1: The TG navigator scenario: specifying conditions for improving traveler risk assessment using biometric-enabled watchlist screening. The TG factors are identified in the causal network for traveler risk assessment using biometric-enabled watchlist and e-ID validation.

\subsection{The TG formalization in terms of probabilities}

Biometric-enabled watchlists are not used in common practice of rapid traveler risk assessment using automated border control except for some pilot projects. The main reason is that contemporary biometric-based profiling technologies significantly decrease the performance of automated gates. Detailed analyses of such scenarios are reported, in particular, in [Do16]. Specifically, technology challenges address the False Match Rate (FMR) and False Non-Match Rate (FNMR). The FMR is the probability that a match is invalid: $\operatorname{Pr}\left(w_{2} \mid m_{1}\right)$, which is the proportion of invalid matches (the traveler is not on the watchlist $W=w_{2}$ ) among all matches (the traveler is matched on the watchlist, either correctly or incorrectly $\left.M=m_{1}\right)$. The FNMR is the probability that a non-match is invalid: $\operatorname{Pr}\left(w_{1} \mid m_{2}\right)$, which is the proportion of invalid non-matches (the traveler is on the watchlist $W=w_{1}$ ) among all non-matches (the traveler is not matched to the watchlist, either correctly or incorrectly $M=m_{2}$ ). Both the FMR and FNMR affect security and privacy: in the case of a false match, an innocent person will be mistakenly directed to manual control; and a false non-match results in entry to a country being granted to a person of interest without manual control. Conceptually, the solution to this problem is known as an improvement to the quality of biometric traits [Do16]. Hence, the FMR and FNMR are indicators of the TG. The problem of bridging the TG can be formulated in terms of the TG navigation over related parameters and indicators.

Definition 1. The TG (Technology Gap) is defined as the goals for the accuracy of various elements of a biometric enabled service required to reach specific goals in the posterior probabilities/belief values for certain target scenarios. In the example covered in this paper, the technology gap refers to the target correctness rates required to satisfy upper bounds on the FMR and FNMR. 
Definition 2. The TG navigator of a given scenario is defined as the process (metric, algorithm) of determining the technology gap that must be spanned to address the scenario.

Definition 3. Bridging the TG is defined as the act of upgrading the existing technology to achieve the accuracy goals.

The TG conditions specification: The threshold $T$, decision strategy $S$, and the number of probes $P$ will be assumed to be arbitrarily fixed.

The TG goal: We will be interested in:

1. $\quad$ the correctness rate for watchlist travelers $x_{1}=\operatorname{Pr}\left(c_{1} \mid w_{1}, T, S, P\right)$, and

2. the correctness rate for non-watchlist travelers $x_{2}=\operatorname{Pr}\left(c_{1} \mid w_{2}, T, S, P\right)$

that will achieve a desired FMR $y_{1}\left(x_{1}, x_{2}\right)=\operatorname{Pr}\left(w_{2} \mid T, S, P, m_{1}\right)$ and a FNMR $y_{2}\left(x_{1}, x_{2}\right)=$ $\operatorname{Pr}\left(w_{1} \mid T, S, P, m_{2}\right)$ of at most $10 \%$. These correctness rates $x_{1}$ and $x_{2}$ establish the TG that must be cleared to achieve the desired FMR and FNMR. The tables below contains the necessary data to calculate $y_{1}, y_{2}$ from $x_{1}, x_{2}$. The uncertainty inference in performed using a software package that is available upon request.

\begin{tabular}{|c||c|c|c||c|c|}
\hline \multirow{2}{*}{$W$} & \multicolumn{2}{|c|}{ Watchlist } & \multicolumn{2}{|c|}{ Correctness } \\
\cline { 3 - 5 } & On List & Off List & & True & False \\
\hline $\operatorname{Pr}(W)$ & 0.0028 & 0.9972 \\
\cline { 3 - 5 } & & $\operatorname{Pr}\left(C \mid w_{1}, T, S, P\right)$ & $x_{1}$ & $1-x_{1}$ \\
$\operatorname{Pr}\left(C \mid w_{2}, T, S, P\right)$ & $x_{2}$ & $1-x_{2}$ \\
\hline
\end{tabular}

\begin{tabular}{|c|c||c|c|}
\hline \multicolumn{2}{|c||}{$\operatorname{Pr}(M \mid W, C)$} & \multicolumn{2}{c|}{ Matching } \\
\hline$W$ & $C$ & Match & Non-match \\
\hline \hline On List & True & 1 & 0 \\
On List & False & 0 & 1 \\
Off List & True & 0 & 1 \\
Off List & False & 1 & 0 \\
\hline
\end{tabular}

The $\left(x_{1}, x_{2}\right)$ pairs of interest are those such that $y_{1}\left(x_{1}, x_{2}\right) \leq 0.1$ and $y_{2}\left(x_{1}, x_{2}\right) \leq 0.1$. The set/region of $\left(x_{1}, x_{2}\right)$ pairs where $y_{1}\left(x_{1}, x_{2}\right) \leq 0.1$ are referred to as $R_{1}$, and the set/region of $\left(x_{1}, x_{2}\right)$ pairs where $y_{2}\left(x_{1}, x_{2}\right) \leq 0.1$ form $R_{2}$. To determine an optimal $x_{1}$ and $x_{2}$, a recursive binary search will be used. The regions $R_{1}$ and $R_{2}$ can be exactly computed by solving a linear system of equations. The table below lists $y_{1}=\operatorname{Pr}\left(w_{2} \mid T, S, P, m_{1}\right)$ and $y_{2}=$ $\operatorname{Pr}\left(w_{1} \mid T, S, P, m_{2}\right)$ given various values for $x_{1}=\operatorname{Pr}\left(c_{1} \mid w_{1}, T, S, P\right)$ and $x_{2}=\operatorname{Pr}\left(c_{1} \mid w_{2}, T, S, P\right)$. Initially, $x_{1}$ and $x_{2}$ are restricted to the set of values $\{0.0,0.5,1.0\}$.

\begin{tabular}{|cc||cc|}
\hline$x_{1}$ & $x_{2}$ & $y_{1}$ & $y_{2}$ \\
\hline 0.00 & 0.00 & 1.000000 & 1.000000 \\
0.00 & 0.50 & 1.000000 & 0.005584 \\
0.00 & 1.00 & $0 / 0$ & 0.002800 \\
0.50 & 0.00 & 0.998598 & 1.000000 \\
0.50 & 0.50 & 0.997200 & 0.002800 \\
\hline
\end{tabular}

\begin{tabular}{|cc||cc|}
\hline$x_{1}$ & $x_{2}$ & $y_{1}$ & $y_{2}$ \\
\hline 0.50 & 1.00 & 0.000000 & 0.001402 \\
1.00 & 0.00 & 0.997200 & $0 / 0$ \\
1.00 & 0.50 & 0.994416 & 0.000000 \\
1.00 & 1.00 & 0.000000 & 0.000000 \\
\hline
\end{tabular}

Decision: We observe that

1. the ranges $[0.00,0.50] \times[0.50,1.00]$ and $[0.50,1.00] \times[0.50,1.00]$ are partially contained by $R_{1}$.

2. the ranges $[0.00,0.50] \times[0.50,1.00]$ and $[0.50,1.00] \times[0.50,1.00]$ are completely contained by $R_{2}$, and $[0.50,1.00] \times[0.00,0.50]$ is partially contained by $R_{2}$.

After a range of interest has been identified as the location of the TG $x_{1}$ and $x_{2}$, the range can be further divided into 4 regions that can be explored recursively. If TG goals $x_{1}$ and $x_{2}$ are chosen from a square that is: 
1. completely contained by $R_{2}$, then the FNMR is guaranteed to be at most 0.1 .

2. partially contained by $R_{2}$, then the FNMR may be at most 0.1 but not guaranteed.

After the TG $x_{1}$ (the desired correctness for subjects on the watchlist) and $x_{2}$ (the desired correctness for ones not on the watchlist) has been chosen to improve the FMR and FNMR to at most $10 \%$, the next problem is improving the existing technology to span the chosen TG. Such improvements may include adjustments to the deep learning approach to train the feature extractor, and hardware and infrastructure improvements such as better lighting etc.

\subsection{The TG formalization in terms of fuzzy probabilities}

The fuzzy probabilities that will be used in this paper are based on [BDT03, Re09]. The fuzzy numbers have the form $(l, c, u)$ where $l$ and $u$ are the lower and upper bounds of the membership function respectively, and $c$ is the center value. Specifically, the membership function $\psi: \mathbb{R} \rightarrow[0,1]$ is triangular: $\psi(x)=0$ for $x \leq l, \frac{x-l}{c-l}$ for $l \leq x \leq c, \frac{u-x}{u-c}$ for $c \leq x \leq u$, and 0 for $u \leq x$. It should also be noted that the lower and upper bounds of the membership function, $l$ and $u$ respectively, may fall outside of the interval [0,1]. In this case, these bounds help to shape the membership function inside of the interval [0,1], but do not enable any point probability to exist outside of the interval $[0,1]$. Using the same TG example, the TG goals, $x_{1}=\operatorname{Pr}\left(c_{1} \mid w_{1}, T, S, P\right)$ and $x_{2}=\operatorname{Pr}\left(c_{1} \mid w_{2}, T, S, P\right)$, and the FMR and FNMR, $y_{1}\left(x_{1}, x_{2}\right)=\operatorname{Pr}\left(w_{2} \mid T, S, P, m_{1}\right)$ and $y_{2}\left(x_{1}, x_{2}\right)=\operatorname{Pr}\left(w_{1} \mid T, S, P, m_{2}\right)$, are now triangular fuzzy numbers. The tables below contain the necessary data to calculate $y_{1}, y_{2}$ from $x_{1}, x_{2}$.

\begin{tabular}{|c||c|c|}
\hline \multicolumn{1}{|c||}{$W$} & \multicolumn{2}{c|}{ Watchlist } \\
\cline { 2 - 3 }$W$ & On List & Off List \\
\hline \hline $\operatorname{Pr}(W)$ & $(0,0.0028,0.1028)$ & $(0.8972,0.9972,1)$ \\
\hline
\end{tabular}

\begin{tabular}{|c||c|c|}
\hline \multicolumn{1}{|c||}{$C$} & \multicolumn{2}{c|}{ Correctness } \\
\cline { 2 - 3 }$C$ & True & False \\
\hline $\operatorname{Pr}\left(C \mid w_{1}, T, S, P\right)$ & $x_{1}$ & $(1,1,1)-x_{1}$ \\
$\operatorname{Pr}\left(C \mid w_{2}, T, S, P\right)$ & $x_{2}$ & $(1,1,1)-x_{2}$ \\
\hline
\end{tabular}

\begin{tabular}{|c|c||c|c|}
\hline \multicolumn{2}{|c||}{$\operatorname{Pr}(M \mid W, C)$} & \multicolumn{2}{c|}{ Matching } \\
\hline$W$ & $C$ & Match & Non-match \\
\hline \hline 'On List' & 'True' & $(1,1,1)$ & $(0,0,0)$ \\
'On List' & 'False' & $(0,0,0)$ & $(1,1,1)$ \\
'Off List' & 'True' & $(0,0,0)$ & $(1,1,1)$ \\
'Off List' & 'False' & $(1,1,1)$ & $(0,0,0)$ \\
\hline
\end{tabular}

\section{Decision:}

1. The $\left(x_{1}, x_{2}\right)$ pairs of interest are those that yield a pair $\left(y_{1}, y_{2}\right)$ that satisfies conditions $f_{1}\left(y_{1}\right)$ and $f_{2}\left(y_{2}\right)$.

2. $f_{1}\left(y_{1}\right)$ is an unknown condition which holds when $y_{1}$ is "small". $f_{2}\left(y_{2}\right)$ is an unknown condition which holds when $y_{2}$ is "small".

3. The set of $\left(x_{1}, x_{2}\right)$ pairs where $f_{1}\left(y_{1}\left(x_{1}, x_{2}\right)\right)$ holds will be referred to as $R_{1}$, and the set of $\left(x_{1}, x_{2}\right)$ pairs where $f_{2}\left(y_{2}\left(x_{1}, x_{2}\right)\right)$ holds will be referred to as $R_{2}$.

Table below lists $y_{1}=\operatorname{Pr}\left(w_{2} \mid T, S, P, m_{1}\right)$ and $y_{2}=\operatorname{Pr}\left(w_{1} \mid T, S, P, m_{2}\right)$ given various values for $x_{1}=\operatorname{Pr}\left(c_{1} \mid w_{1}, T, S, P\right)$ and $x_{2}=\operatorname{Pr}\left(c_{1} \mid w_{2}, T, S, P\right) . x_{1}$ and $x_{2}$ are restricted to the set of values $\{(0.0,0.0,0.5),(0.0,0.5,1.0),(0.5,1.0,1.0)\}$. These 3 values are fuzzy versions of the values $\{0.0,0.5,1.0\}$. After the TG has been determined, the same approaches can be used to bridge the TG as when point probabilities were used. However, because fuzzy probabilities are being used, the requirements set by the TG are not as strict as when point probabilities are being used. It is also important note that using fuzzy probabilities 


\begin{tabular}{|c|c||cc|}
\hline$x_{1}$ & $x_{2}$ & $y_{1}$ & $y_{2}$ \\
\hline$(0.00,0.00,0.50)$ & $(0.00,0.00,0.50)$ & $(0.43,1.00,2.23)$ & $(0.00,1.00,+\infty)$ \\
$(0.00,0.00,0.50)$ & $(0.00,0.50,1.00)$ & $(0.00,1.00,+\infty)$ & $(0.00,0.01,+\infty)$ \\
$(0.00,0.00,0.50)$ & $(0.50,1.00,1.00)$ & $(0.00,0 / 0,+\infty)$ & $(0.00,0.00,0.23)$ \\
$(0.00,0.50,1.00)$ & $(0.00,0.00,0.50)$ & $(0.41,1.00,2.23)$ & $(0.00,1.00,+\infty)$ \\
$(0.00,0.50,1.00)$ & $(0.00,0.50,1.00)$ & $(0.00,1.00,+\infty)$ & $(0.00,0.00,+\infty)$ \\
$(0.00,0.50,1.00)$ & $(0.50,1.00,1.00)$ & $(0.00,0.00,+\infty)$ & $(0.00,0.00,0.23)$ \\
$(0.50,1.00,1.00)$ & $(0.00,0.00,0.50)$ & $(0.41,1.00,2.23)$ & $(0.00,0 / 0,+\infty)$ \\
$(0.50,1.00,1.00)$ & $(0.00,0.50,1.00)$ & $(0.00,0.99,+\infty)$ & $(0.00,0.00,+\infty)$ \\
$(0.50,1.00,1.00)$ & $(0.50,1.00,1.00)$ & $(0.00,0.00,+\infty)$ & $(0.00,0.00,0.12)$ \\
\hline
\end{tabular}

to navigate the TG is not the same as sensitivity analysis. Sensitivity analysis aims to determine the impact of uncertainty, which is distinct from navigating the TG which aims to compute the TG while accounting for uncertainty.

\section{Summary and conclusion}

The complexity of biometric-enabled systems for rapid individual risk assessment in masstransit hubs is the main motivation for the emerging development of the TG navigator. In a complex security system, the designed and the achieved performance can be significantly different. To avoid or mitigate this effect, advanced modeling techniques such machine reasoning should be chosen. The proposed TG navigator addresses this problem. The framework of the TG navigator includes advances in recognition, probabilistic modeling, computational intelligence, and sensitivity analysis. Our development and experimental testing of the TG navigator results in the following conclusions:

1. The TG navigator provides an assessment of risk for the scenarios in which performance requirements are at the critical level of technological possibilities.

2. The TG navigator helps identify the conditions for bridging the TGs when designing the systems under technological constraints. The mechanism for such analysis is the machine reasoning based on a tailored analysis of probability distributions, through Bayesian inference.

The uncertainty metrics that were the subject of the examples are point probability distributions and fuzzy probability distributions. It is important to note that other uncertainty metrics such as probability intervals and Dempster-Shafer models can be utilized.

\section{References}

[Ab17] Abouelenien, Mo.; Pérez-Rosas, V.; Mihalcea, R.; Burzo, M.: Detecting deceptive behavior via integration of discriminative features from multiple modalities. IEEE Transactions on Information Forensics and Security, 12(5):1042-1055, 2017.

[ANH18] Almudhahka, N.; Nixon, M.; Hare, J.: Semantic Face Signatures: Recognizing and Retrieving Faces by Verbal Descriptions. IEEE Transactions on Information Forensics and Security, 13(3):706-716, 2018.

[BDT03] Baldwin, J.; Di Tomaso, E.: Inference and learning in fuzzy Bayesian networks. In: IEEE International Conference on Fuzzy Systems. volume 1, pp. 630-635, 2003. 
8 S. Eastwood, K. Lai, S. Yanushkevich, R. Guest and V. Shmerko

[Bi17] Biggio, B.; Fumera, G.; Marcialis, G.; Roli, F.: Statistical meta-analysis of presentation attacks for secure multibiometric systems. IEEE transactions on pattern analysis and machine intelligence, 39(3):561-575, 2017.

[BP16] Borgonovo, E.; Plischke, E.: Sensitivity analysis: a review of recent advances. European Journal of Operational Research, 248(3):869-887, 2016.

[Ca17] Castillo, E.; Grande, Z.; Mora, E.; Lo, H.; Xu, X.: Complexity reduction and sensitivity analysis in road probabilistic safety assessment Bayesian network models. Computer-Aided Civil and Infrastructure Engineering, 32(7):546-561, 2017.

[CHR15] Chatterjee, S.; Hora, S.; Rosoff, H.: Portfolio analysis of layered security measures. Risk Analysis, 35(3):459-475, 2015.

[Do16] Donida Labati, R.; Genovese, A.; Muñoz, E.; Piuri, V.; Scotti, F.; Sforza, G.: Biometric Recognition in Automated Border Control: A Survey. ACM Computing Surveys, 49(2):A:1-A:39, 2016

[EU14] EU European Commission B-1049: , Technical Study on Smart Borders, 2014.

[GNQ17] Grother, P.; Ngan, M.; Quinn, G.: , Face in video evaluation (FIVE) Face recognition of non-cooperative subjects, Report 817, 2017.

[Ha05] Hartman, J.; Atkinson, D.; Lind, M.; Maughan, A.; Kelly, J.: Technology Gap Analysis for the Detection of Process Signatures Using Less Than Remote Methods. Technical report, Pacific Northwest National Laboratory (PNNL), Richland, WA (US), 2005.

[In14] International Air Transport Association: , Checkpoint of the future. Executive summary. 4th Proof., 2014.

[JR15] Jain, A.; Ross, A.: Bridging the gap: from biometrics to forensics. Phil. Trans. R. Soc. B, 370(1674):20140254, 2015

[KS18] K., Ueda; S., Kurahashi: Agent-based self-service technology adoption model for airtravelers: Exploring best operational practices. Frontiers in Physics, 6:1-14, 2018.

[La17] Lai, K.; Yanushkevich, S.; Shmerko, V.; Eastwood, S.: Bridging the gap between forensics and biometric-enabled watchlists for e-borders. IEEE Computational Intelligence Magazine, 12(1):16-28, 2017.

[Le13] Lee, Y.; Filliben, J.; Micheals, R.; Phillips, J.: Sensitivity analysis for biometric systems: A methodology based on orthogonal experiment designs. Computer Vision and Image Understanding, 117(5):532-550, 2013.

[Li14] Liu, Y.; Hansen, M.; Gupta, G.; Malik, W.; Jung, Y.: Predictability impacts of airport surface automation. Transportation Research Part C: Emerging Technologies, 44:128-145, 2014.

[Nu14] Nuppency, M.: , Automated border control - state of play and latest developments, Federal Office for Information Security, 2014.

[Pa08] Palmer, A.: Criteria to evaluate automated personal identification mechanisms. Computers \& Security, 27(7-8):260-284, 2008.

[Re09] Ren, J.; Jenkinson, I.; Wang, J.; Xu, DL.; Yang, JB.: An offshore risk analysis method using fuzzy Bayesian network. Journal of Offshore Mechanics and Arctic Engineering, 131(4):041101, 2009.

[RZ14] Rajoub, B.; Zwiggelaar, R.: Thermal facial analysis for deception detection. IEEE transactions on information forensics and security, 9(6):1015-1023, 2014. 
8 S. Eastwood, K. Lai, S. Yanushkevich, R. Guest and V. Shmerko

[Bi17] Biggio, B.; Fumera, G.; Marcialis, G.; Roli, F.: Statistical meta-analysis of presentation attacks for secure multibiometric systems. IEEE transactions on pattern analysis and machine intelligence, 39(3):561-575, 2017.

[BP16] Borgonovo, E.; Plischke, E.: Sensitivity analysis: a review of recent advances. European Journal of Operational Research, 248(3):869-887, 2016.

[Ca17] Castillo, E.; Grande, Z.; Mora, E.; Lo, H.; Xu, X.: Complexity reduction and sensitivity analysis in road probabilistic safety assessment Bayesian network models. Computer-Aided Civil and Infrastructure Engineering, 32(7):546-561, 2017.

[CHR15] Chatterjee, S.; Hora, S.; Rosoff, H.: Portfolio analysis of layered security measures. Risk Analysis, 35(3):459-475, 2015.

[Do16] Donida Labati, R.; Genovese, A.; Muñoz, E.; Piuri, V.; Scotti, F.; Sforza, G.: Biometric Recognition in Automated Border Control: A Survey. ACM Computing Surveys, 49(2):A:1-A:39, 2016

[EU14] EU European Commission B-1049: , Technical Study on Smart Borders, 2014.

[GNQ17] Grother, P.; Ngan, M.; Quinn, G.: , Face in video evaluation (FIVE) Face recognition of non-cooperative subjects, Report 817, 2017.

[Ha05] Hartman, J.; Atkinson, D.; Lind, M.; Maughan, A.; Kelly, J.: Technology Gap Analysis for the Detection of Process Signatures Using Less Than Remote Methods. Technical report, Pacific Northwest National Laboratory (PNNL), Richland, WA (US), 2005.

[In14] International Air Transport Association: , Checkpoint of the future. Executive summary. 4th Proof., 2014.

[JR15] Jain, A.; Ross, A.: Bridging the gap: from biometrics to forensics. Phil. Trans. R. Soc. B, 370(1674):20140254, 2015

[KS18] K., Ueda; S., Kurahashi: Agent-based self-service technology adoption model for airtravelers: Exploring best operational practices. Frontiers in Physics, 6:1-14, 2018.

[La17] Lai, K.; Yanushkevich, S.; Shmerko, V.; Eastwood, S.: Bridging the gap between forensics and biometric-enabled watchlists for e-borders. IEEE Computational Intelligence Magazine, 12(1):16-28, 2017.

[Le13] Lee, Y.; Filliben, J.; Micheals, R.; Phillips, J.: Sensitivity analysis for biometric systems: A methodology based on orthogonal experiment designs. Computer Vision and Image Understanding, 117(5):532-550, 2013.

[Li14] Liu, Y.; Hansen, M.; Gupta, G.; Malik, W.; Jung, Y.: Predictability impacts of airport surface automation. Transportation Research Part C: Emerging Technologies, 44:128-145, 2014.

[Nu14] Nuppency, M.: , Automated border control - state of play and latest developments, Federal Office for Information Security, 2014.

[Pa08] Palmer, A.: Criteria to evaluate automated personal identification mechanisms. Computers \& Security, 27(7-8):260-284, 2008.

[Re09] Ren, J.; Jenkinson, I.; Wang, J.; Xu, DL.; Yang, JB.: An offshore risk analysis method using fuzzy Bayesian network. Journal of Offshore Mechanics and Arctic Engineering, 131(4):041101, 2009.

[RZ14] Rajoub, B.; Zwiggelaar, R.: Thermal facial analysis for deception detection. IEEE transactions on information forensics and security, 9(6):1015-1023, 2014. 